PROCEEDINGS OF THE

AMERICAN MATHEMATICAL SOCIETY

Volume 130, Number 10, Pages 2931-2939

S 0002-9939(02)06535-8

Article electronically published on April 10, 2002

\title{
ASYMPTOTICS OF SOBOLEV EMBEDDINGS AND SINGULAR PERTURBATIONS FOR THE $p$-LAPLACIAN
}

\author{
MANUEL DEL PINO AND CÉSAR FLORES \\ (Communicated by David S. Tartakoff) \\ To the memory of Carlos Cid
}

\begin{abstract}
We consider the best constant $S\left(\Omega_{\lambda}\right)$ for the embedding of $W^{1, p}\left(\Omega_{\lambda}\right)$ into $L^{q}\left(\Omega_{\lambda}\right)$ where $1<p<2, p<q<\frac{N p}{N-p}$. Here $\Omega_{\lambda}=\lambda \Omega$ with $\Omega$ a smooth, bounded domain in $\mathbb{R}^{n}$ and $\lambda$ a large positive number. It is proven by the validity of the expansion$$
S\left(\Omega_{\lambda}\right)=S\left(\mathbb{R}_{+}^{n}\right)-\lambda^{-1} \gamma \max _{x \in \partial \Omega} H(x)+o\left(\lambda^{-1}\right),
$$

as $\lambda \rightarrow \infty$, where $\gamma$ is a positive constant depending on $p, q$ and $N$. The behavior of associated extremals, which satisfy an equation involving the $p$ Laplacian operator, is also analyzed.
\end{abstract}

\section{IntRoduCtion AND STATEMENT OF MAIN RESUlTS}

Let $\Omega$ be a bounded domain in $\mathbb{R}^{N}, N \geq 2$, with $\partial \Omega$ smooth. Let $p>1$ and denote $p^{*}=\frac{N p}{N-p}$ if $p<N, p^{*}=+\infty$ otherwise. It is well known that for any $1<q<p^{*}$ the Sobolev embedding of $W^{1, p}(\Omega)$ into $L^{q}(\Omega)$ holds, namely there exists a positive constant $S=S(p, q, \Omega)$ such that

$$
S\|u\|_{L^{q}(\Omega)}^{p} \leq\|u\|_{W^{1, p}(\Omega)}^{p}
$$

for all $u \in W^{1, p}(\Omega)$. The best constant for this embedding is the largest $S$ for which the above relation holds, namely the number $S(\Omega)$ defined as

$$
S(\Omega)=\inf _{u \in W^{1, p}(\Omega) \backslash\{0\}} \frac{\int_{\Omega}|\nabla u|^{p}+|u|^{p}}{\left(\int_{\Omega}|u|^{q}\right)^{\frac{p}{q}}} .
$$

This embedding is compact, which implies the existence of extremals for it, namely, functions $u$ at which this infimum is achieved.

Let us fix $p$ and $q$ as above, and a bounded smooth domain $\Omega$. For a large positive number $\lambda$ we consider the family of expanding domains

$$
\Omega_{\lambda}=\lambda \Omega=\{\lambda x \mid x \in \Omega\} .
$$

Our purpose in this paper is to describe the asymptotic behavior as $\lambda \rightarrow+\infty$ of the best constants $S\left(\Omega_{\lambda}\right)$ as well as that of the associated family of extremals $u_{\lambda}$.

Received by the editors May 1, 2001.

2000 Mathematics Subject Classification. Primary 35J20; Secondary 35B40.

This work was supported by grants Fondecyt Lineas Complementarias 8000010, DIUC 200.015.015-1.0, ECOS/CONICYT, and FONDAP. 
In what follows we shall denote by $u_{\lambda}$ an extremal normalized so that the relation

$$
\int_{\Omega_{\lambda}}\left|\nabla u_{\lambda}\right|^{p}+\left|u_{\lambda}\right|^{p}=\int_{\Omega_{\lambda}}\left|u_{\lambda}\right|^{q}
$$

holds. Then the Euler-Lagrange equation satisfied by $u_{\lambda}$ becomes

$$
\left\{\begin{aligned}
\Delta_{p} u_{\lambda}-\left|u_{\lambda}\right|^{p-2} u_{\lambda}+\left|u_{\lambda}\right|^{q-2} u_{\lambda}=0 & \text { in } \quad \Omega_{\lambda} \\
u>0 \text { in } \Omega_{\lambda}, \frac{\partial u}{\partial \eta}=0 & \text { on } \quad \partial \Omega_{\lambda} .
\end{aligned}\right.
$$

Here $\Delta_{p}$ stands for the $p$-Laplacian operator, $\Delta_{p} u=\operatorname{div}\left(|\nabla u|^{p-2} \nabla u\right)$. Note that $u_{\lambda}$ is one-signed in $\Omega$. Indeed, $\left|u_{\lambda}\right|$ is also a minimizer of the Raleigh quotient above, hence also a solution to (1.2). Regularity theory for the $p$-Laplacian (see [5, 11]) applies to yield that $\left|u_{\lambda}\right|$ is actually of class $C^{1, \alpha}(\Omega)$. Then the strong maximum principle proved in 12 implies that $\left|u_{\lambda}\right|$ does not vanish in $\Omega$, and therefore $u_{\lambda}$ is one-signed. Henceforth we will assume $u_{\lambda}>0$ in $\Omega$.

Since $\Omega_{\lambda}$ expands toward entire space or to a half-space depending on the choice of origin, it is natural to relate the behavior of $S\left(\Omega_{\lambda}\right)$ and $u_{\lambda}$ with best constant and extremals of the Sobolev embedding in $\mathbb{R}^{N}$,

$$
S\left(\mathbb{R}^{N}\right)=\inf _{u \in W^{1, p}\left(\mathbb{R}^{N}\right) \backslash\{0\}} \frac{\int_{\mathbb{R}^{N}}|\nabla u|^{p}+|u|^{p}}{\left(\int_{\mathbb{R}^{N}}|u|^{q}\right)^{\frac{p}{q}}} .
$$

A concentration-compactness argument along the lines of [1, [7] shows that this infimum is achieved. Modulo normalization, extremals are positive solutions of the equation

$$
\begin{array}{cl}
\Delta_{p} w-|w|^{p-2} w+|w|^{q-2} w=0 & \text { in } \mathbb{R}^{N}, \\
w(x) \rightarrow 0 & \text { as }|x| \rightarrow+\infty .
\end{array}
$$

It has been established in [2] that for $1<p<2$, positive solutions of (1.3) are radially symmetric around some point. Moreover, from a recent result in [10], the radial solution around the origin is unique. We shall denote it by $w_{*}=w_{*}(|x|)$ in what follows. Let us consider the half-space $\mathbb{R}_{+}^{N}=\left\{\left(x^{\prime}, x_{N}\right) \mid x_{N}>0\right\}$. The best constants of $\mathbb{R}_{+}^{N}$ and $\mathbb{R}^{N}$ relate as

$$
S\left(\mathbb{R}_{+}^{N}\right)=2^{-\frac{q-p}{q}} S\left(\mathbb{R}^{N}\right) .
$$

Corresponding extremals are positive solutions of the problem

$$
\begin{array}{ll}
\Delta_{p} w-|w|^{p-2} w+|w|^{q-2} w=0 & \text { in } \mathbb{R}_{+}^{N}, \\
w(x) \rightarrow 0 \text { as }|x| \rightarrow+\infty, \quad \frac{\partial w}{\partial x_{N}}=0 & \text { on } \partial \mathbb{R}_{+}^{N} .
\end{array}
$$

Even extension of such a solution to entire space corresponds to a solution of problem (1.3). It is natural to suspect that $S\left(\Omega_{\lambda}\right)$ converges to the corresponding quantity for the half-space, and $u_{\lambda}$ to an associated extremal. Our principal result states that when $1<p<2, S\left(\Omega_{\lambda}\right)$ indeed approaches $S\left(\mathbb{R}_{+}^{N}\right)$, corrected by a negative factor of the maximum mean curvature of $\partial \Omega$.

Theorem 1.1. Assume that $1<p<2$ and that $p<q<\frac{N p}{N-p}$. There is a constant $\gamma=\gamma(p, q, N) 0$ such that the following expansion holds:

$$
S\left(\Omega_{\lambda}\right)=S\left(\mathbb{R}_{+}^{N}\right)-\lambda^{-1} \gamma \max _{x \in \partial \Omega} H(x)+o\left(\lambda^{-1}\right),
$$


as $\lambda \rightarrow+\infty$. Here $H(x)$ denotes the mean curvature of the boundary at the point $x$. Moreover, there exist points $x^{\lambda} \in \partial \Omega$ such that

$$
\sup _{y \in \Omega_{\lambda}}\left|u_{\lambda}(y)-w_{*}\left(\left|y-\lambda x^{\lambda}\right|\right)\right| \rightarrow 0
$$

and

$$
H\left(x^{\lambda}\right) \rightarrow \max _{x \in \partial \Omega} H(x)
$$

as $\lambda \rightarrow \infty$.

The radial symmetry of the extremals of $S\left(\mathbb{R}^{N}\right)$ plays a crucial role in the proof of the theorem. For $p>2$ the same result would hold if such a fact was true. The constant $\gamma$ above is given explicitly as follows:

$$
\gamma=\frac{2 p}{q} \frac{N-1}{N+1} \omega_{N} \omega_{N-1} \int_{0}^{\infty}\left|w_{*}^{\prime}(r)\right|^{p} r^{N} d r \int_{0}^{\infty} w_{*}(r)^{q} r^{N-1} d r .
$$

For $p=2$, these facts have been known since the works by Lin Ni and Takagi, and $\mathrm{Ni}$ and Takagi [6, 8, 9]. The proof devised in those works does not apply in the current situation. Strong use of linearity of the differential operator, as well as certain nondegeneracy properties of the linearized equation around $w_{*}$ only known for $p=2$, is used. A different proof of those results was found in [4]. We borrow ideas from that work in the proof of Theorem 1.1. See also [3] for a related result involving trace embeddings and $p=2$.

\section{Preliminaries}

Let us consider the best Sobolev constant $S\left(\Omega_{\lambda}\right)$ given by (1.1) for the embedding of $W^{1, p}\left(\Omega_{\lambda}\right)$ into $L^{q}\left(\Omega_{\lambda}\right)$. It is convenient for our purposes to obtain a further characterization of this value and its extremals in terms of the energy functional

$$
J_{\lambda}(u)=\frac{1}{p} \int_{\Omega_{\lambda}}\left(|\nabla u|^{p}+u^{p}\right) d x-\frac{1}{q} \int_{\Omega_{\lambda}} u_{+}^{q} d x .
$$

It is standard to check that nontrivial critical points of $J_{\lambda}$ in $W^{1, p}\left(\Omega_{\lambda}\right)$ correspond precisely to the positive solutions of problem (1.2). Let us consider the number

$$
c_{\lambda} \equiv \inf _{\substack{u \in W^{1, p}\left(\Omega_{\lambda}\right) \\ u \neq 0}} \sup _{t>0} J_{\lambda}(t u) .
$$

It is easy to see that if $u_{+} \neq 0$, the function $t \mapsto J_{\lambda}(t u)$ has a maximum $t=\bar{t}>0$ which is its unique critical point. Then $\bar{t} u \in M_{\lambda}$, where

$$
M_{\lambda}=\left\{u \in W^{1, p}\left(\Omega_{\lambda}\right) / u \neq 0, \int_{\Omega_{\lambda}}|\nabla u|^{p}+u^{p}=\int_{\Omega_{\lambda}} u_{+}^{q}\right\}
$$

is the so-called Nehari's manifold of $J_{\lambda}$. It follows from this fact that

$$
c_{\lambda}=\inf _{u \in M_{\lambda}} J_{\lambda}(u) \text {. }
$$

Since all nontrivial solutions of (1.2) lie in $M_{\lambda}$, the above number is called the least energy value for $J_{\lambda}$ and a solution $u$ of (1.2) with $J_{\lambda}(u)=c_{\lambda}$, a least energy solution. These solutions and extremals of $S\left(\Omega_{\lambda}\right)$ are related in the following way: if $u$ is a least energy solution, then it is an extremal of $S\left(\Omega_{\lambda}\right)$. Reciprocally, if $\bar{u} \geq 0$ 
minimizes the Raleigh quotient (1.1), then $u=t \bar{u}$ is a least energy solution of (1.2) where

$$
t^{q-p}=\frac{\int_{\Omega_{\lambda}}|\nabla \bar{u}|^{p}+\bar{u}^{p}}{\int_{\Omega_{\lambda}} \bar{u}^{q}} .
$$

In fact we always have the exact relation

$$
c_{\lambda}=\left(\frac{1}{p}-\frac{1}{q}\right) S\left(\Omega_{\lambda}\right)^{\frac{q}{q-p}} .
$$

As we have mentioned, for $\Omega_{\lambda}$ bounded the compactness of the associated embedding yields the existence of extremals for $S\left(\Omega_{\lambda}\right)$ and correspondingly of critical points of $J_{\lambda}$ at level $c_{\lambda}$.

Now, we establish an $L^{\infty}$ estimate for solutions of (1.2) in terms of their energy values.

Lemma 2.1. Let $u$ be a solution of (1.2). Then there are constants $B=B(\Omega, p, N)$ and $\theta=\theta(\Omega, p, N)$, independent of $1 \leq \lambda<\infty$, such that

$$
\|u\|_{\infty} \leq B J_{\lambda}(u)^{\theta} .
$$

Proof. We consider a positive solution $u$ of (1.2). Let us multiply (1.2) by $u^{\alpha p+1-p}$, where $\alpha \geq 1$. Integrating over $\Omega_{\lambda}$, we find that

$$
\frac{\alpha p+1-p}{\alpha^{p}} \int_{\Omega_{\lambda}}\left|\nabla u^{\alpha}\right|^{p}+\int_{\Omega_{\lambda}} u^{\alpha p}=\int_{\Omega_{\lambda}} u^{\alpha p+q-p} .
$$

Noticing that $\frac{1}{2 \alpha^{p-1}} \leq \min \left\{1, \frac{\alpha p+1-p}{2 \alpha^{p}}\right\}$, we get

$$
\frac{1}{2 \alpha^{p-1}} \int_{\Omega_{\lambda}}\left|\nabla u^{\alpha}\right|^{p}+u^{\alpha p} \leq \int_{\Omega_{\lambda}} u^{\alpha p+q-p} .
$$

Sobolev's inequality applied for $v=u^{\alpha}$ yields

$$
\left(\int_{\Omega_{\lambda}} u^{\alpha p^{*}}\right)^{\frac{p}{p^{*}}} \leq C \alpha^{p-1} \int_{\Omega_{\lambda}} u^{q+\alpha p-p}
$$

where, we recall, $p^{*}=\frac{N p}{N-p}$ and the constant $C$ is independent of $\lambda$. Next, we consider the sequence of positive numbers $\alpha_{j}, j=0,1,2, \ldots$, defined inductively as

$$
q+\alpha_{0} p-p=p^{*}, \quad q+\alpha_{j+1} p-p=\alpha_{j} p^{*}, \quad \forall j=0,1, \ldots,
$$

or, explicitly,

$$
\alpha_{j}=\frac{\left(p^{*} / p\right)^{j+1}\left(p^{*}-q\right)+q-p}{p^{*}-p} .
$$

Note that, by (2.7) with $\alpha=1$,

$$
\left(\int_{\Omega_{\lambda}} u^{p^{*}}\right)^{p / p^{*}} \leq C\left(\frac{1}{p}-\frac{1}{q}\right)^{-1} J_{\lambda}(u)
$$

where $J_{\lambda}$ is the energy functional given by (2.1). Now we will construct a suitable sequence of positive numbers $M_{j}$ such that

$$
\int_{\Omega_{\lambda}} u^{q+\alpha_{j} p-p} \leq M_{j}, \quad \forall j .
$$


Inequality (2.10) gives us $M_{0}$. Assuming that (2.11) holds, we have by (2.7) and (2.9) that

$$
\int_{\Omega_{\lambda}} u^{q+\alpha_{j+1} p-p} \leq\left(C \alpha_{j}^{p-1} \int_{\Omega_{\lambda}} u^{q+\alpha_{j} p-p}\right)^{p^{*} / p} \leq\left(C \alpha_{j}^{p-1} M_{j}\right)^{p^{*} / p} .
$$

(2.11) then holds for $M_{j}$ defined as

$$
M_{0}=\left(C\left(\frac{1}{p}-\frac{1}{q}\right)^{-1} J_{\lambda}(v)\right)^{p^{*} / q}, M_{j+1}=\left(C \alpha_{j}^{p-1} M_{j}\right)^{p^{*} / q} \forall j \geq 0 .
$$

From these relations, the explicit form of the $\alpha_{j}$ 's given by (2.9), and a straightforward computation, we find constants $C_{1}, C_{2}$ depending only on $C, p, q, N$ such that

$$
M_{j} \leq \exp \left(C_{1} \log M_{0}\right) \exp \left(C_{2} \alpha_{j-1}\left(1+\log M_{0}\right)\right)
$$

and using relations (2.7)-(2.9),

$$
\|v\|_{L^{\alpha_{j} p^{*}}\left(\Omega_{\lambda}\right)} \leq\left(\exp \left(C_{1} \log M_{0}\right)\right)^{\frac{p}{\alpha_{j} p^{*}}} \exp \left(C_{2} / p^{*}\left(1+\log M_{0}\right)\right) .
$$

Letting $j \rightarrow \infty$ we obtain the result.

Now we claim that $J_{\lambda}\left(u_{\lambda}\right)$ is uniformly bounded independently of $\lambda$. In fact, let us consider $w_{\lambda}^{z}(x)=w_{*}(\lambda(x-z))$, where $z \in \partial \Omega$ and $w_{*}$ is the radial least energy solution of equation (1.3). Using the definition of $c_{\lambda}$ and a direct computation we then find that

$$
c_{\lambda} \leq \sup _{t>0} J_{\lambda}\left(t w_{\lambda}^{z}\right) \leq \frac{c_{*}}{2}+o(1)
$$

where $c_{*}$ is the corresponding energy of $w_{*}$ in the whole space $\mathbb{R}^{N}$, namely $c_{*} / 2$ is that in the half-space. As a consequence, the above lemma yields a uniform $L^{\infty}$ estimate for the solutions $u_{\lambda}$. Moreover, from the $C^{1, \alpha}$ estimates found in [5] and [1], we have the validity of the following

Lemma 2.2. There is a constant $C$ independent of $\lambda$ such that for any least energy solution $u_{\lambda}$ of (1.2)

$$
\left\|u_{\lambda}\right\|_{C^{1, \alpha}\left(\Omega_{\lambda}\right)} \leq C
$$

We end this section by establishing uniform exponential decay on the least energy solutions $u_{\lambda}$ to (1.2).

Lemma 2.3. There exist positive constants $\alpha, \beta$ such that

$$
u_{\lambda}(y) \leq \alpha \exp \left(-\beta\left|y-y^{\lambda}\right|\right)
$$

for all $y \in \Omega_{\lambda}$ and $\lambda$ sufficiently large. Here $y^{\lambda}$ denotes any maximum point of $u_{\lambda}$.

Proof. First, we will see that the functions $u_{\lambda}$ decay uniformly at infinity, namely that given $\varepsilon>0$ there is an $R>0$ such that $u_{\lambda}(y)<\varepsilon$ whenever $\left|y-y^{\lambda}\right|>R$. By contradiction, let us assume that for some $\varepsilon>0$ there are sequences $\lambda_{n} \rightarrow \infty$ and $y^{n} \in \bar{\Omega}_{\lambda_{n}}$ such that $\left|y^{\lambda_{n}}-y^{n}\right| \rightarrow \infty$ and $u_{\lambda_{n}}\left(y_{n}\right) \geq \epsilon$. We claim that under these conditions,

$$
\liminf _{n \rightarrow \infty} J_{\lambda_{n}}\left(u_{n}\right) \geq c_{*}
$$

which is a contradiction with relation (2.16). Since $u_{\lambda_{n}}$ is uniformly bounded in $C^{1, \alpha}$, we may assume passing to a subsequence that $u_{\lambda_{n}}\left(y^{\lambda_{n}}+y\right) \rightarrow u(y)$ uniformly 
over compacts, where, thanks to our contradiction assumption, $u$ is a positive solution of (1.3) or, after a rotation and translation, a positive solution of (1.4), depending on whether the distance $\operatorname{dist}\left(y_{\lambda_{n}}, \partial \Omega_{\lambda_{n}}\right) \rightarrow+\infty$ or remained uniformly bounded. Let us introduce the notation

$$
J_{\Lambda}(v)=\frac{1}{p} \int_{\Lambda}|\nabla v|^{p}+v^{p}-\frac{1}{q} \int_{\Lambda} v^{q} .
$$

Then, given $\delta>0$ we have that for all sufficiently large $R$,

$$
\lim _{n \rightarrow \infty} J_{B_{R}\left(y^{\lambda_{n}}\right) \cap \Omega_{\lambda_{n}}}\left(u_{\lambda_{n}}\right) \geq \frac{c_{*}}{2}-\delta .
$$

Similarly, for all large $R$,

$$
\lim _{n \rightarrow \infty} J_{B_{R}\left(y^{n}\right) \cap \Omega_{\lambda_{n}}}\left(u_{\lambda_{n}}\right) \geq \frac{c_{*}}{2}-\delta .
$$

Let us consider $R>0$ and a smooth cut-off function $\eta_{R}^{n}$ such that $\eta_{R}^{n} \equiv 0$ on $B_{R-1}\left(y^{\lambda_{n}}\right) \cup B_{R-1}\left(y^{n}\right), 0 \leq \eta_{R}^{n} \leq 1, \eta_{R}^{n} \equiv 1$ on $\mathbb{R}^{N} \backslash\left(B_{R}\left(y^{\lambda_{n}}\right) \cup B_{R}\left(y^{n}\right)\right)$, and $\left|\nabla \eta_{R}^{n}\right| \leq C, C$ independent of $R$ and $n$.

We use $w_{n}=\eta_{R}^{n} u_{\lambda_{n}}$ as a test function for $J_{\lambda_{n}}^{\prime}\left(u_{\lambda_{n}}\right)=0$ to obtain

$$
0=J_{\lambda_{n}}^{\prime}\left(u_{\lambda_{n}}\right) w_{n}=E_{n}+p J_{\mathbb{R}^{N} \backslash B_{R}\left(y^{\lambda_{n}}\right) \cup B_{R}\left(y^{n}\right)}\left(u_{\lambda_{n}}\right)+\int_{\Omega_{\lambda_{n}}} g_{n}
$$

where $g_{n}=\left(\frac{p}{q}-1\right) u_{\lambda_{n}}^{q} \eta_{R}^{n} \leq 0$ and $E_{n}$ is given by

$$
E_{n}=\int_{A_{R}^{n}}\left|\nabla u_{\lambda_{n}}\right|^{p-2} \nabla u_{\lambda_{n}} \nabla\left(\eta_{R}^{n} u_{\lambda_{n}}\right)+\eta_{R}^{n} u_{\lambda_{n}}^{p}
$$

where $A_{R}^{n}=\left\{y \in \Omega_{\lambda_{n}} / R-1<\left|y-y^{\lambda_{n}}\right|<R\right.$ or $\left.R-1<\left|y-y^{n}\right|<R\right\}$. The convergence of $u_{\lambda_{n}}$ in the $C^{1}$-sense over compacts around $y_{n}$ and $y^{\lambda_{n}}$ to functions in $W^{1, p}\left(\mathbb{R}^{N}\right)$ implies that for $R>0$ sufficiently large $\lim _{n \rightarrow \infty}\left|E_{n}\right| \leq \delta$. It follows that for large enough $R, J_{\mathbb{R}^{N} \backslash B_{R+1}\left(y^{\lambda_{n}}\right) \cup B_{R+1}\left(y^{n}\right)}\left(u_{\lambda_{n}}\right) \geq-\delta$. Using this together with relations (2.19) and (2.20), (2.17) follows.

The desired exponential decay will be a consequence of the following

Claim. There exists $R_{0}>0$ and $\nu_{0}>0$ such that for all $R>R_{0}$

$$
\sup _{\left|y-y^{\lambda}\right| \geq R} u_{\lambda}(y) \geq 2 \sup _{\left|y-y^{\lambda}\right| \geq R+\nu_{0}} u_{\lambda}(y)
$$

for all $\lambda$ sufficiently large.

By contradiction, let us assume that there exist sequences $R_{n} \rightarrow \infty, \bar{\lambda}_{n} \rightarrow \infty$, $\nu_{n} \rightarrow \infty$ and $\bar{y}^{n} \in \Omega_{\bar{\lambda}_{n}}$ with $\left|\bar{y}^{n}-y^{\bar{\lambda}_{n}}\right| \geq R_{n}+\nu_{n}$ such that $u_{\bar{\lambda}_{n}}\left(\bar{y}^{n}\right)=\mu_{n}>\frac{1}{2} M_{n}$, where

$$
M_{n}=\sup _{\left|y-y^{\bar{\lambda}_{n}}\right|>R_{n}} u_{\bar{\lambda}_{n}}(y) .
$$

From the uniform decay established above, we see that $M_{n}, \mu_{n} \rightarrow 0$. Let us set $v_{n}(y)=\mu_{n}^{-1} u_{\bar{\lambda}_{n}}\left(y+\bar{y}_{n}\right)$. Then $v_{n}$ is bounded, $v_{n}(0)=1$ and satisfies

$$
\Delta_{p} v_{n}-\left(1-\left|u_{\lambda_{n}}\left(y+\bar{y}_{n}\right)\right|^{q-p}\right) v_{n}^{p-1}=0 \quad \text { in } \Omega_{\bar{\lambda}_{n}}-\bar{y}_{n},
$$

with $\frac{\partial v_{n}}{\partial \nu}=0$ on the boundary. Letting $n \rightarrow \infty$ we obtain a contradiction since $v_{n}$ converges locally uniformly to a positive bounded solution $v$ of the limiting problem $\Delta_{p} v-v^{p-1}=0, v(0)=1$, in the entire space $\mathbb{R}^{N}$ or in the half-space $\mathbb{R}_{+}^{N}$, with $\frac{\partial v}{\partial \nu}=0$ on $\mathbb{R}^{N-1}$. This object does not exist. Indeed, let us consider a sequence 
$x_{n}$ with $v\left(x_{n}\right) \rightarrow \sup _{x \in \mathbb{R}^{N}} v(x) \geq 1$, and define $w_{n}(x)=v\left(x_{n}+x\right)$. Since $w_{n}$ is uniformly bounded, it follows that $w_{n}$ also has a uniform $C^{1, \alpha}$ bound. Thus passing to a subsequence, we may assume that $w_{n} \rightarrow w$ uniformly over compacts, where again $w$ solves $\Delta_{p} w-w^{p-1}=0$, but now $w(0)=\max _{x \in \mathbb{R}^{N}} w(x)$. The strong maximum principle in [12] yields a contradiction. Thus, the claim holds and we have the uniform exponential decay of the functions $u_{\lambda}$. By standard arguments involving local elliptic estimates, we obtain the validity of the same property for the derivatives of $u_{\lambda}$.

Remark 2.1. Let $y^{\lambda}$ be a point where $u_{\lambda}$ reaches its maximum value. Then the above proof also shows that $\operatorname{dist}\left(y^{\lambda}, \partial \Omega_{\lambda}\right)$ must remain bounded, for otherwise we would end up in the limit with an energy of a level at least $c^{*}$.

\section{Proof of Theorem 1.1}

Let $u_{\lambda}$ be a positive least energy solution of (1.2), that is a solution with $\lambda\left(u_{\lambda}\right)=$ $c_{\lambda}$. Let $y^{\lambda}$ be a point in $\bar{\Omega}_{\lambda}$ where $u_{\lambda}$ maximizes, and $x^{\lambda} \in \partial \Omega$ is the closest point in $\partial \Omega$ to $\lambda^{-1} y^{\lambda}$. From Remark 2.1, we then see that $\left|\lambda x^{\lambda}-y^{\lambda}\right|$ remains uniformly bounded. Passing to a subsequence, we assume that $x^{\lambda} \rightarrow \bar{x} \in \partial \Omega$.

Let us set $v_{\lambda}(y)=u_{\lambda}\left(\lambda x_{\lambda}+y\right)$. Then $v_{\lambda}$ converges in the $W^{1, p}$-sense to $w_{*}$. Moreover, for certain positive constants $a$ and $b$ we have $v_{\lambda}(y) \leq a e^{-b|y|}$.

After a rotation and a translation $\lambda$-dependent we may also assume that $x^{\lambda}=0$ and that $\Omega$ can be described in a fixed neighborhood $V$ of $\bar{x}$ as the set $\left\{\left(x^{\prime}, x_{N}\right) \mid x_{N}\right.$ $\left.>G_{\lambda}\left(x^{\prime}\right)\right\}$ where $G_{\lambda}$ is smooth, $G_{\lambda}(0)=0$ and $G_{\lambda}^{\prime}(0)=0$. Further, we may also assume that $G_{\lambda}$ converges locally in a $C^{2}$-sense to $G$, a corresponding parametrization at $\bar{x}$.

Let us also set $\widetilde{\Omega}_{\lambda}=\Omega_{\lambda}-\lambda x_{\lambda}$. From the variational characterization of $c_{\lambda}$, we have that

$$
J_{\lambda}\left(u_{\lambda}\right) \geq J_{\lambda}\left(t u_{\lambda}\right)=J_{\widetilde{\Omega}_{\lambda}}\left(t v_{\lambda}\right)
$$

for all $t>0$. Let us define the function $\tilde{v}_{\lambda}$ on $\lambda\left(\mathbb{R}_{+}^{N} \cap V\right)$ as $\tilde{v}_{\lambda}\left(y^{\prime}, y_{N}\right)=v_{\lambda}\left(y^{\prime}, y_{N}\right)$ if $G_{\lambda}\left(y^{\prime} / \lambda\right)>0$ and $\tilde{v}_{\lambda}\left(y^{\prime}, y_{N}\right)=v_{\lambda}\left(y^{\prime}, \lambda G_{\lambda}\left(y^{\prime} / \lambda\right)\right)$ if $G_{\lambda}\left(y^{\prime} / \lambda\right) \leq 0$. Then

$$
J_{\widetilde{\Omega}_{\lambda}}\left(t v_{\lambda}\right) \geq J_{\mathbb{R}_{+}^{N} \cap V_{\lambda}}\left(t \tilde{v}_{\lambda}\right)+J_{\left(\widetilde{\Omega}_{\lambda} \cap V_{\lambda}\right) \backslash \mathbb{R}_{+}^{N}}\left(t v_{\lambda}\right)-J_{\left(\mathbb{R}_{+}^{N} \cap V_{\lambda}\right) \backslash \tilde{\Omega}_{\lambda}}\left(t \tilde{v}_{\lambda}\right) .
$$

Let us choose $t=t_{\lambda}$ so that $J_{\mathbb{R}_{+}^{N} \cap V_{\lambda}}\left(t \tilde{v}_{\lambda}\right)$ maximizes in $t$. Then, by definition of the number $c\left(\mathbb{R}_{+}^{N}\right)=c_{*} / 2$ and the exponential decay of $v_{\lambda}$, one gets that

$$
J_{\mathbb{R}_{+}^{N} \cap V_{\lambda}}\left(t_{\lambda} \tilde{v}_{\lambda}\right) \geq \frac{c_{*}}{2}+O\left(e^{-2 \lambda \alpha}\right)
$$

for some $\alpha>0$. Again using the exponential decay of $v_{\lambda}$ we obtain

$$
\begin{aligned}
& I_{1}=-J_{\left(\widetilde{\Omega}_{\lambda} \cap V_{\lambda}\right) \backslash \mathbb{R}_{+}^{N}}\left(t_{\lambda} v_{\lambda}\right) \\
& =-\int_{B_{\lambda}} d y^{\prime} \int_{\lambda G_{\lambda}\left(y^{\prime} / \lambda\right)_{-}}^{0}\left(\frac{1}{p}\left|\nabla v_{\lambda}\right|^{p}+\frac{1}{p} v_{\lambda}^{p}-\frac{1}{q} v_{\lambda}^{q}\right)\left(y^{\prime}, y_{N}\right) d y_{N}+O\left(e^{-2 \lambda \alpha /}\right),
\end{aligned}
$$

where $B_{\lambda}=\left\{\left|y^{\prime}\right|<\lambda \delta\right\}$. Similarly, we find that

$$
\begin{aligned}
& I_{2}=J_{\left(\mathbb{R}_{+}^{N} \cap V_{\lambda}\right) \backslash \widetilde{\Omega}_{\lambda}}\left(t_{\lambda} \tilde{v}_{\lambda}\right) \\
& =\int_{B_{\lambda}} d y^{\prime} \int_{0}^{\lambda G_{\lambda}\left(y^{\prime} / \lambda\right)_{+}}\left(\frac{1}{p}\left|\nabla v_{\lambda}\right|^{p}+\frac{1}{p} v_{\lambda}^{p}-\frac{1}{q} v_{\lambda}^{q}\right)\left(y^{\prime}, G_{\lambda}\left(y^{\prime} / \lambda\right)\right) d y_{N}+O\left(e^{-2 \lambda \alpha}\right) .
\end{aligned}
$$


Here we have denoted $a_{+}=\max \{a, 0\}, a_{-}=\min \{a, 0\}$. Now we note that $v_{\lambda} \rightarrow w$ $C^{1}$-locally with uniform exponential decay. Then since $G_{\lambda}(0)=0$ and $G_{\lambda}^{\prime}(0)=0$ and $G_{\lambda}$ converges in a $C^{2}$ local sense to $G$, an application of dominated convergence yields

$$
\begin{aligned}
\lim _{\lambda \rightarrow \infty} \lambda\left(I_{1}+I_{2}\right) & =\sum_{i, j=1}^{N-1} \int_{\mathbb{R}^{N-1}} G_{i j}^{\prime \prime}(0) y_{i}^{\prime} y_{j}^{\prime}\left(\frac{1}{p}\left|\nabla w_{*}\right|^{p}+\frac{1}{p} w_{*}^{p}-\frac{1}{q} w_{*}^{q}\right)\left(y^{\prime}, 0\right) d y^{\prime} \\
& =\tilde{\gamma} \sum_{i=1}^{N-1} G_{i i}^{\prime \prime}(0)=\tilde{\gamma} H(\bar{x}) .
\end{aligned}
$$

Here

$$
\tilde{\gamma}=(N-1) \omega_{N-1} \int_{0}^{\infty}\left\{\frac{1}{p}\left(\left|w_{*}^{\prime}(r)\right|^{p}+w_{*}(r)^{p}\right)-\frac{1}{q} w_{*}(r)^{q}\right\} d r .
$$

Using $r^{2} w_{*}^{\prime}(r)$ as a test function in the equation satisfied by $w_{*}$, one obtains

$$
\tilde{\gamma}=2 \frac{N-1}{N+1} \omega_{N-1} \int_{0}^{\infty} r^{N}\left|w_{*}^{\prime}(r)\right|^{p} d r
$$

We conclude that

$$
c_{\lambda} \geq \frac{c_{*}}{2}-\lambda^{-1} \gamma H(\bar{x})+o\left(\lambda^{-1}\right) .
$$

On the other hand, using a computation along the same lines as above, refining estimate (2.16) yields

$$
c_{\lambda} \leq \sup _{t>0} J_{\lambda}\left(t w_{\lambda}^{z}\right)=\frac{c_{*}}{2}-\lambda^{-1} \gamma H(z)+o\left(\lambda^{-1}\right),
$$

for any $z \in \partial \Omega$. Here $w_{\lambda}^{z}=w_{*}\left(\lambda\left(x-x_{\lambda}\right)\right)$.

Combining these two estimates directly provides assertions (1) and (2) of the theorem, since in particular we conclude $H(\bar{x}) \geq H(z)$ for all $z \in \partial \Omega$. Finally, relation (2.4) yields the desired expansion (1.5) for $S\left(\Omega_{\lambda}\right)$, and the proof of Theorem 1.1 is concluded.

\section{ACKNOWLEDGEMENT}

We would like to thank Patricio Felmer who suggested the study of this problem to us and provided us with useful references. We also thank the referee for a careful reading of the paper and useful suggestions.

\section{REFERENCES}

[1] H. Berestycki, P.L. Lions, Nonlinear scalar field equations. II. Existence of infinitely many solutions. Arch. Rational Mech. Anal. 82 (1983), 4, 347-375. MR 84h:35054b

[2] L. Damascelli, F. Pacella, M. Ramaswamy. Symmetry of ground states of $p$-Laplace equations via the moving plane method. Arch. Ration. Mech. Anal. 148 (1999), no. 4, 291-308. MR 2000j:35080

[3] M. del Pino, C. Flores, Asymptotic behavior of best constants and extremals for trace embeddings in expanding domains. Comm. Partial Differential Equations, to appear.

[4] M. del Pino, P. Felmer. Spike-layered solutions of singularly perturbed elliptic problems in a degenerate setting. Indiana Univ. Math. J. 48 (1999), 883-898. MR 2001b:35027

[5] E. Di Benedetto, $C^{1+\alpha}$ local regularity of weak solutions of degenerate elliptic equations, Nonlinear Analysis 7 (1983), 827-850. MR 85d:35037

[6] C.S. Lin, W.M. Ni, I. Takagi. Large amplitude stationary solutions to a chemotaxis system. J. Differential Equations 72 (1988), 1-27. MR 89e:35075 
[7] P.L. Lions, The concentration-compactness principle in the calculus of variations. The locally compact case. II. Ann. Inst. H. Poincaré Anal. Non Linéaire 1 (1984), 4, 223-283. MR 87e:49035b

[8] W.M. Ni, I. Takagi. On the shape of least-energy solutions to a semilinear Neumann problem. Comm. Pure Appl. Math. 44 (1991), 819-851. MR 92i:35052

[9] W.M. Ni, I. Takagi. Locating the peaks of least-energy solutions to a semilinear Neumann problem. Duke Math. J. 70 (1993), 247-281. MR 94h:35072

[10] J. Serrin, M. Tang. Uniqueness of ground states for quasilinear elliptic equations. Indiana Univ. Math. J. 49 (2000), no. 3, 897-923.

[11] P. Tolksdorf. Regularity for a more general class of quasilinear elliptic equations. J. Differential Equations 51 (1984), no. 1, 126-150. MR 85g:35047

[12] J.L. Vázquez. A strong maximum principle for some quasilinear elliptic equations. Appl. Math. Optim. 12 (1984), 191-202. MR 86m:35018

Departamento de Ingeniería Matemática and Centro de Modelamiento Matemático (UMr2071 CNRS-UChile), Universidad de Chile, Casilla 170, Correo 3, Santiago, Chile E-mail address: delpino@dim.uchile.cl

Departamento de Matemáticas, FCFm Universidad de Concepción, Casilla 160-C, Concepción, Chile

E-mail address: cflores@dim.uchile.cl 